International Journal of Engineering \& Technology, $7(2.33)(2018) 670-672$
International Journal of Engineering \& Technology
WPC
Website www.sciencepubco.com/index.php/IJET
Research paper

\title{
Amplify-forward and decode-forward: a novel power allocation technique for MIMO cognitive radio network
}

\author{
M. Monisha ${ }^{1}$, M. Meena ${ }^{1}$, C. Sharanya ${ }^{1}$, T. Jaya $^{1}$ \\ ${ }^{1}$ Department of Electronics and Communication and Engineering, School of Engineering, Vels Institute of Science Technology \& Ad- \\ vanced Studies (VISTAS), Vels University, Chennai, Tamil Nadu, India \\ *Corresponding author E-mail: jaya.se@velsuniv.ac.in
}

\begin{abstract}
Nowadays transmitting data through wireless communication channel requires more power than the wired communication channel. In other words any communication through wireless medium will consume more power. Since power is a limited source, it is hard to conserve it or reduce its usage and this leads to the concept of "Efficient power allocation scheme for MIMO (Multi Input Multi Output) cognitive radio" system. The proposed system uses two algorithms namely On/Off Based Scheduling (OOBS) and Selective Based Scheduling (SBS) in Amplify and Forward (AAF) and Decode and Forward (DAF) techniques. OOBS algorithm is used for efficient allocation of the input power to each user's Signal to Noise Ratio (SNR) value. The proposed algorithm provides better power utilization with low bit error rate $(9.53 \times 10-7)$.
\end{abstract}

Keywords: MIMO;AF; DF; Efficient Power Allocation.

\section{Introduction}

In wireless network the major factor is the demand of high data rate and high speed over wireless communication system. MIMO OFDM is the promising technique to achieving the high data rate in wireless communication channel. Single Input Single Output (SISO) communication systems are not much efficient so new systems are developed like MIMO which are much more efficient than the SISO. The power requirement is much higher than the SISO system and error rate also will be high. So the cognitive radio network helps to allocate the power in efficient manner and reduce the bit error rate.

Cognitive radio network take decisions using the information about the RF environment through some models and past experiences. Spectrum sharing and wireless communications are the main objectives of cognitive radio network. There are two types:

1) Primary

2) Secondary

Where licensed persons are primary user and unlicensed persons is secondary user. Nowadays the power allocation in MIMO cognitive network has to be efficient, since the power is limited resource.

There are many techniques for power allocation in MIMO cognitive radio system [1] [2], such as fixed allocation, dynamic allocation, MA (Margin Adaptive) rate adaptive. The existing techniques for power allocation in MIMO networks are not effective in allocating the power efficiently. Hence we are proposing a new algorithm based on OOBS and SBS scheduling using hybrid AAF and DAF relay techniques.

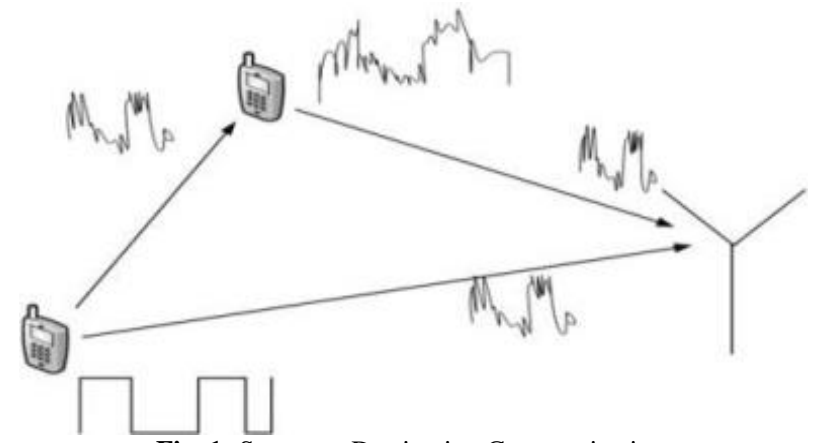

Fig. 1: Source to Destination Communication.

\section{Methodology}

The proposed method for allocating power efficiently is based on AAF and DAF relay techniques using OBS and SBS algorithm. The input signal is the OFDM modulated signals [2]. In MIMO [3] [4] cognitive radio network different users will have different SNR values. The OFDM modulated MIMO signals are transmitted through a Rayleigh fading channel i. e (20 KHz).

\subsection{OOBS and SBS}

The OOBS technique is used in this method allows only those signals, which are above the predefined SNR values. The SNR of the each user compared with the predefined value and acceptable signals only pass through the channel. The SBS scheduling will allow the unacceptable signals if some part of the channels is ideal. Theses election techniques will continue until the channel bandwidth utilization is full.The power is efficiently allocated based on the SNR value of each user and given by the formula shown below 
Power $=(1 / \mathrm{SNR} *$ initial power/no of user $)$

(1)

The user with lowest SNR is allocated maximum power and the user with highest SNR allocated minimum power. The hybrid $\mathrm{AAF}$ and DAF relays are used for reducing the power needed for direct source to destination communication.

The MRC technique is used for selecting the appropriate relay techniques[5]. The MRC technique is used for achieving maximum SNR values by combining the individual SNR values.

\subsection{AAF and DAF}

The AAF technique is implemented between the relay to destination. The main aim of this technique is used to simply amplify the received signals [6] [7]. And it is used to boost up the received week signals.

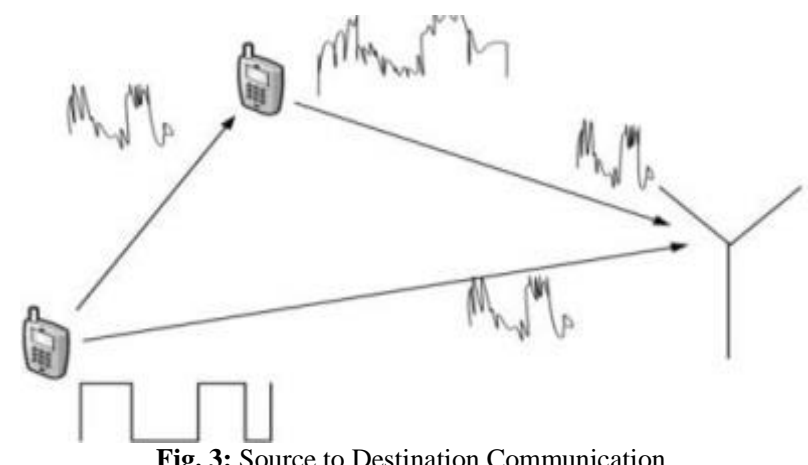

3) The DAF technique is implemented between source and relay. The main aim of this technique is used to encode the received signals and also used to correct the error if any present in the received signals[8][9]. DAF technique helps to reduce the errors and decode the received signals and retransmit to the next level.

Figure 2 represents the block diagram of AAF, DAF and hybrid relay technique. An MIMO cognitive radio network is designed the source and destination nodes are fixed along with some intermediate nodes. The intermediate nods are acting as a relay. Here the signals are chosen based on the SNR value. If the signals SNR value is greater than $20 \mathrm{~dB}$ then those signals are acceptable, otherwise those signals are non-acceptable signals. If the selected signals bandwidth is equal to the channel bandwidth then all the signals are going through that channel to next level. If the selected signals bandwidth is not equal to the channel bandwidth then the channel bandwidth is not properly utilized. By using the SBS scheduling technique the un-utilized bandwidth are used in a proper manner. The un-selected signals are listed in descending order and the most priority signals like high SNR signals are selected and passed to the channel. The signal selections are based on the SNR value and channel bandwidth. The steps to be followed in the proposed technique are

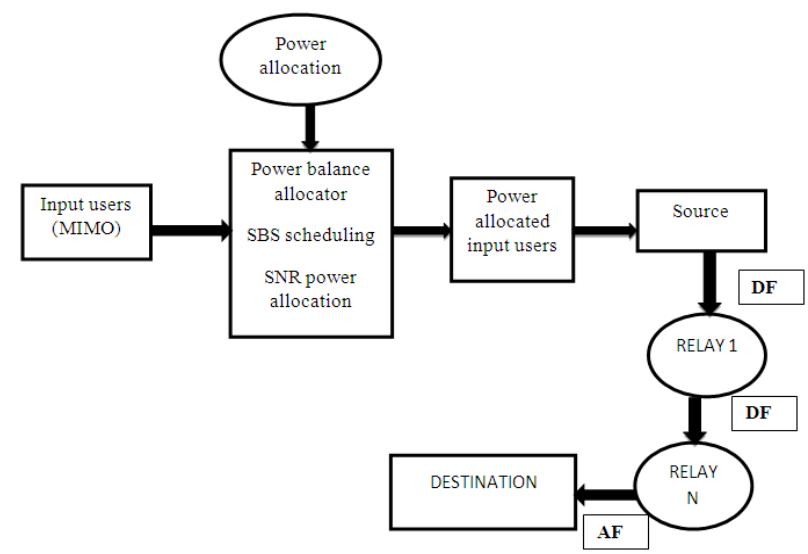

Fig. 2: Block Diagram for Proposed Technique.
Step 1: Random SNR Generation.

Step 2: Check the SNR value and choose the acceptable signals.

Step 3: Power allocation for each user depending the SNR value

Step 4: Source to relay communication $-\mathrm{DAF}$

Relay to destination communication $-\mathrm{AAF}$

Step 5: Final output.

The below graph is explain the signal selection among the all other signals.

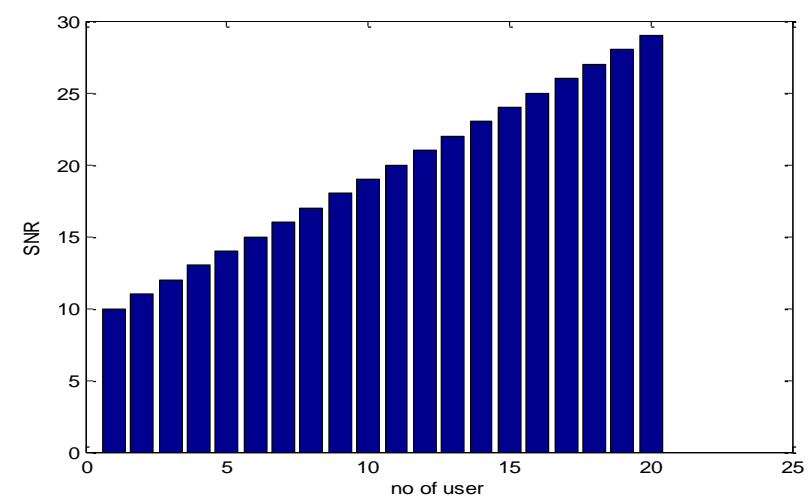

Fig. 3: Input Signals (No of Users vs SNR).

From the figure $3 \mathrm{X}$-axis represents no of users, $\mathrm{y}$-axis represents SNR value. From the graph we can easily find the each user's SNR value.

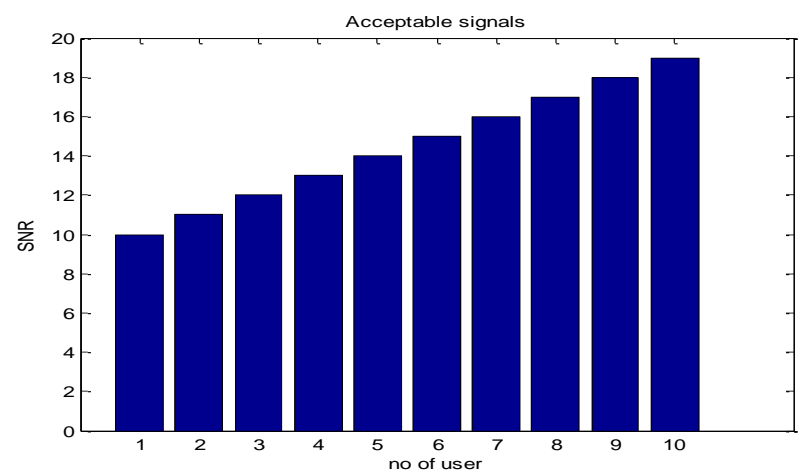

Fig. 4: Acceptable Signals (No of Users vs. SNR).

From the figure 4 can easily find the acceptable signals that can get eligible to go through the channel. If these signals are equal to the channel bandwidth means the power is allocated to these signals depending upon the SNR value. After allocating the power the relay selection is done. There are many relay selection techniques is already used, but here MRC technique is used for relay selection. The congestion free shortest path from source to destination is found by using the routing algorithm. We design a network with 6 nodes. The source ID is node 1 and destination ID is node 6 . From source to relay DAF (decode and forward) technique is chosen. This will helps to reduce the error if any presents in the transmitted signals, and retransmit to the next relay or destination. If the next node is relay means DAF only choose else if the next node is destination means AAF is chosen. AAF is mainly used to amplify the received signals. 


\section{Software simulation}

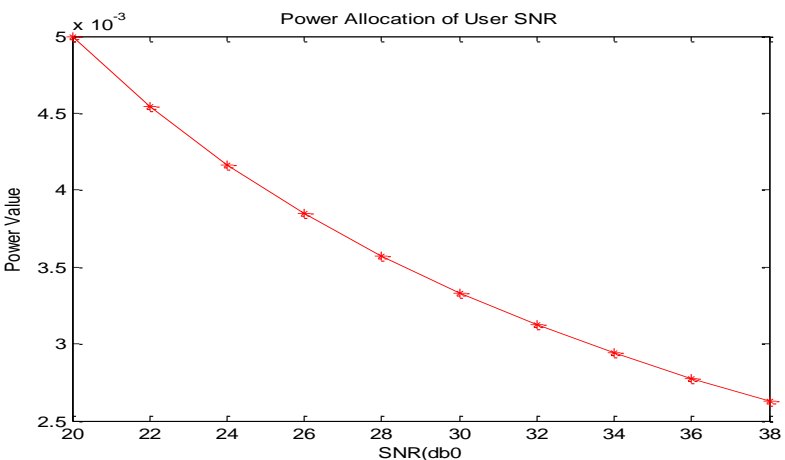

Fig. 5: SNR vs. Power Value.

From the figure 5 represents the power allocation between the all users depending upon the each user's SNR value. In that X-axis represents the SNR value and Y-axis represents the power value. From our proposed method the total number of user is ten, among these 10 users the power allocated depending on the SNR value. I.e. User 1 has low SNR value so the high power is allocated for the first user. Similarly the power is allocated for all the remaining users. User 10 having high SNR value so the less power allocated to that user.

Table 1: SNR vs Power Value

\begin{tabular}{lll} 
& Table 1: SNR vs Power Value \\
\hline S.NO/ USER & SNR $(\mathrm{dB})$ & POWER VALUE \\
\hline 1 & 20 & $5 \times 10^{-3}$ \\
2 & 22 & $4.5 \times 10^{-3}$ \\
3 & 24 & $4.2 \times 10^{-3}$ \\
4 & 26 & $3.8 \times 10^{-3}$ \\
5 & 28 & $3.5 \times 10^{-3}$ \\
6 & 30 & $3.4 \times 10^{-3}$ \\
7 & 32 & $3.2 \times 10^{-3}$ \\
8 & 34 & $3.0 \times 10^{-3}$ \\
9 & 36 & $2.7 \times 10^{-3}$ \\
10 & 38 & $2.56 \times 10^{-3}$ \\
\hline
\end{tabular}

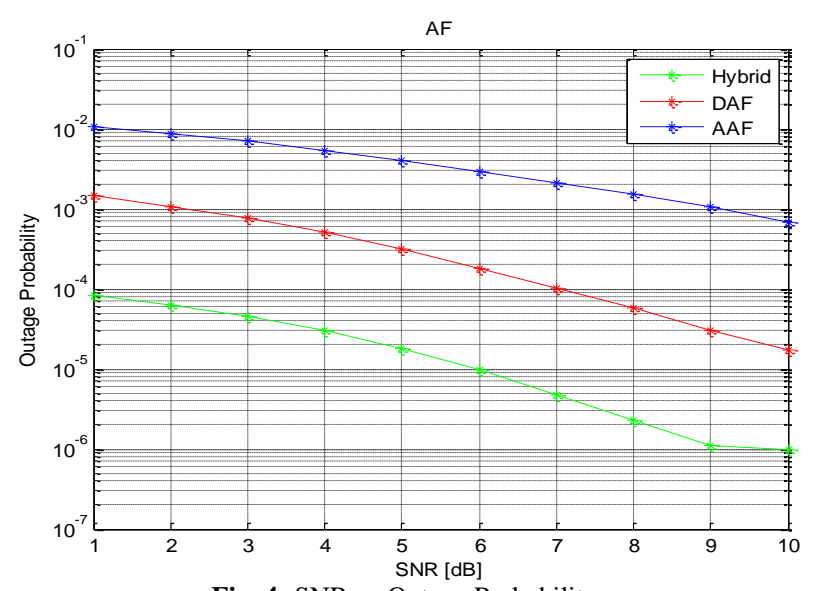

Fig. 4: SNR vs Outage Probability.

From the figure six $\mathrm{X}$-axis represents the SNR value for each users and $\mathrm{Y}$-axis represents the outage probability. In that figure, AAF has high Bit error rate compared to DAF. DAF is less BER when compared to AAF. If we are using AAF for entire communication means BER will very high, and if we are using DF only between the source to destination means it gives less BER compared to AAF. So we implemented Hybrid AAF and DAF technique between source and destination. Now we can see from the graph the BER is reduced compare with both AAF and DAF.

Table 2: SNR vs. BER for AF, DF, and Hybrid

\begin{tabular}{lllll}
\hline S. NO & SNR $(\mathrm{dB})$ & \multicolumn{3}{l}{ Bit Error Rate } \\
\hline & & AF & DF & HYBRID \\
1 & 1 & 0.01047 & 0.001381 & 0.0001016 \\
2 & 2 & 0.008666 & 0.001035 & $7.057 \times 10^{-5}$ \\
\hline
\end{tabular}

\begin{tabular}{lllll}
\hline 3 & 3 & 0.006961 & 0.000762 & $4.945 \times 10^{-5}$ \\
4 & 4 & 0.005385 & 0.0005138 & $3.102 \times 10^{-5}$ \\
5 & 5 & 0.003999 & 0.0003259 & $1.742 \times 10^{-5}$ \\
6 & 6 & 0.002912 & 0.0001924 & $8.984 \times 10^{-6}$ \\
7 & 7 & 0.002084 & 0.0001073 & $4.401 \times 10^{-6}$ \\
8 & 8 & 0.001507 & $6.117 \times 10^{-5}$ & $2.135 \times 10^{-6}$ \\
9 & 9 & 0.001048 & $3.073 \times 10^{-5}$ & $9.54 \times 10^{-7}$ \\
10 & 10 & 0.000765 & $1.953 \times 10^{-5}$ & $9.53 \times 10^{-7}$ \\
\hline
\end{tabular}

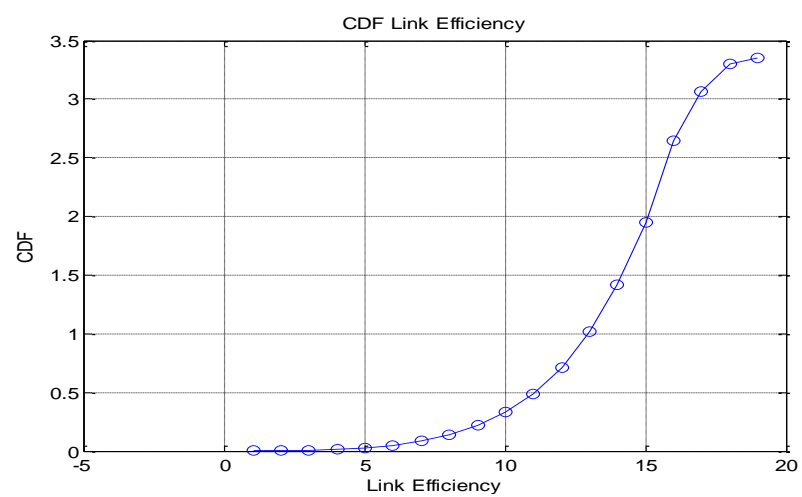

Fig. 7: CDF vs Link Efficiency.

Figure7 represents the link efficiency of the all users.

\section{Conclusion}

In this paper the power allocation for each user is analyzed depending upon the SNR value of the users and the input $1 \mathrm{~dB}$ power is efficiently utilized by all users, and also BER was reduced by the help of AAF and DAF technique. The proposed technique uses two algorithms; they are namely OOBS and SBS in AAF and DAF techniques. ON OFF BASE SCHEDULING (OOBS) algorithm is used efficiently to allocate the input power to each user's SNR value, the proposed algorithm provides high power utilization with low bit error rate.

\section{Acknowledgement}

I wish to express my sincere thanks to Dr. V. Rajendran, Head of Department of Electronics and Communication Engineering, Vels University for his good counsel, encouragement, valuable suggestions and support rendered at all times during my research work, valuable guidance and the facilities provided to me.

\section{References}

[1] L. Fu, Y. J. A. Zhang, and J. Huang, "Energy efficient transmissions in MIMO cognitive radio networks," IEEE J. Sel. Areas Commun., vol. 31, no. 11, pp. 2420-2431, 2013.

[2] W. Bocquet, "A Power Allocation Scheme for MIMO OFDM Systems," vol. 0022, no. 1, pp. 8-11.

[3] J. Shu and W. Guo, "Efficient Bandwidth and Power Allocation Algorithms for Multiuser MIMO-OFDM Systems *," vol. 2009, no. September, pp. 504-510, 2009.

[4] Y. Zou, X. Wang, and W. Shen, "Optimal relay selection for physical-layer security in cooperative wireless networks," IEEE J. Sel. Areas Commun., vol. 31, no. 10, pp. 2099-2111, 2013.

[5] V. K. Shah and A. P. Gharge, "A Review on Relay Selection Techniques in Cooperative Communication," vol. 2, no. 5, pp. 65-69, 2012.

[6] A. I. Akin and H. Ilhan, "RELAY SELECTION FOR AF BASED COOPERATIVE NETWORKS," vol. 19, no. 19, pp. 26-29, 2015.

[7] P. Cheng, R. Deng, and J. Chen, "Energy-efficient cooperative spectrum sensing in sensor-aided cognitive radio networks," IEEE Wirel. Commun, vol. 19, no. 6, pp. 100-105, 2012.

[8] G. Levin and S. Loyka, "Amplify-and-Forward versus Decode-andForward Relaying: Which is Better?" pp. 123-126, 2012.

[9] P. Zhang, J. Yuan, J. Chen, J. Wang, and J. Yang, “Analyzing Amplify-and-Forward and Decode-and-Forward Cooperative Strategies in Wyner s Channel Model." 